\title{
An Unusual Case of Severe Preeclampsia in the Postpartum Period with Hemoperitoneum
}

\author{
${ }^{1}$ Michelle Loh, ${ }^{2}$ Rajeswari Kathirvel
}

\section{ABSTRACT}

Background: HELLP syndrome, consisting of hemolysis, elevated liver enzymes, and low platelet count, is a rare complication of occurring in 10 to $20 \%$ of women with severe preeclampsia and in 0.2 to $0.6 \%$ of total pregnancies. Delivery is the only definitive treatment, with maternal condition usually improving in the postpartum period.

Hemoperitoneum in a woman with preeclampsia is very rare, usually secondary to hepatic hematoma with rupture. While the prevalence of intrahepatic hematoma in these women has been reported to be as high as 39\%, capsular rupture is rare, occurring up to $12 \%$ in women with capsular hematoma, carrying with it high mortality for both the mother and fetus.

Aim: We report an unusual case of hemoperitoneum secondary to liver hematoma in severe preeclampsia in the postpartum period that was conservatively managed leading to good maternal outcome.

Case report: We report a case of postpartum onset of severe preeclampsia in a 25 -year-old woman complicated by hepatic dysfunction with coagulopathy and hemoperitoneum. This was secondary to a contained subcapsular hematoma and was conservatively managed resulting in a good outcome.

Conclusion: Prompt recognition with careful clinical examination, laboratory investigations and imaging, supportive treatment, and early fetal delivery remain the cornerstone in the management. Although reaching the diagnosis was initially challenging in our case study, both mother and baby had a good outcome with an appropriate supportive treatment.

Clinical significance: Most cases with liver hematomas may lead to hepatic rupture with hemodynamic instability, necessitating an aggressive operative approach. However, conservative management with close monitoring of hemodynamic status can be undertaken if the patient is hemodynamically stable with good outcome, as illustrated in this case.

Keywords: Hemoperitoneum, Postpartum, Preeclampsia, Pregnancy.

How to cite this article: Loh M, Kathirvel R. An Unusual Case of Severe Preeclampsia in the Postpartum Period with Hemoperitoneum. J South Asian Feder Obst Gynae 2017;9(4):363-366.

\footnotetext{
${ }^{1}$ Resident, ${ }^{2}$ Consultant

1,2Department of Obstetrics and Gynecology, KK Women's and Children's Hospital, Singapore

Corresponding Author: Michelle Loh, Resident, Department of Obstetrics and Gynecology, KK Women's and Children's Hospital, Singapore, Phone: +90885639, e-mail: michelle.loh@ mohh.com.sg
}

\author{
Source of support: Nil
}

Conflict of interest: None

Date of received: 5 October 2017

Date of acceptance: 7 December 2017

Date of publication: January 2018

\section{BACKGROUND}

HELLP syndrome, consisting of hemolysis, elevated liver enzymes, and low platelet count, is a rare complication of severe preeclampsia. It was first reported by Weinstein in $1982 .{ }^{1}$ It occurs in 10 to $20 \%$ of women with severe

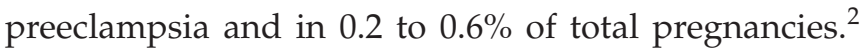
Delivery of the baby is the only definitive treatment, with the maternal condition usually improving in the postpartum period. Risk factors include multiparity, advanced maternal age, and previous personal and family history of preeclampsia/HELLP syndrome. ${ }^{3}$

The overall mortality rate for women with HELLP syndrome is $1.1 \%,{ }^{4}$ and up to $38 \%$ experience adverse maternal outcomes including disseminated intravascular coagulation (DIVC), placental abruption, acute renal failure, pulmonary edema, and subcapsular hematoma. ${ }^{5}$ Perinatal deaths have been reported in about 7.7 to $60 \%$ of cases. $^{6}$

Hemoperitoneum in a woman with preeclampsia is very rare. This is usually secondary to hepatic hematoma with rupture. While the prevalence of intrahepatic hematoma in women with HELLP syndrome has been reported to be as high as 39\%, ${ }^{7}$ capsular rupture is rare, occurring in 1 in 40,000 to 1 in 250,000 deliveries $^{8}$ and 0.53 to $2 \%$ of women with HELLP syndrome. ${ }^{8}$ It occurs in about $12 \%$ of women with capsular hematoma, ${ }^{7}$ with high mortality rates for both the mother (17\%) and the fetus (38\%). ${ }^{9}$ Prompt recognition and management are critical to reduce maternal and fetal morbidity and mortality.

We report a case of severe preeclampsia in the postpartum period complicated by subcapsular hematoma with hepatic dysfunction, coagulopathy, and hemoperitoneum.

\section{CASE REPORT}

A 25-year-old lady attended KK Women's and Children's Hospital $(\mathrm{KKH})$ for routine antenatal care at 34 weeks of gestation in her second pregnancy. She had one previous 


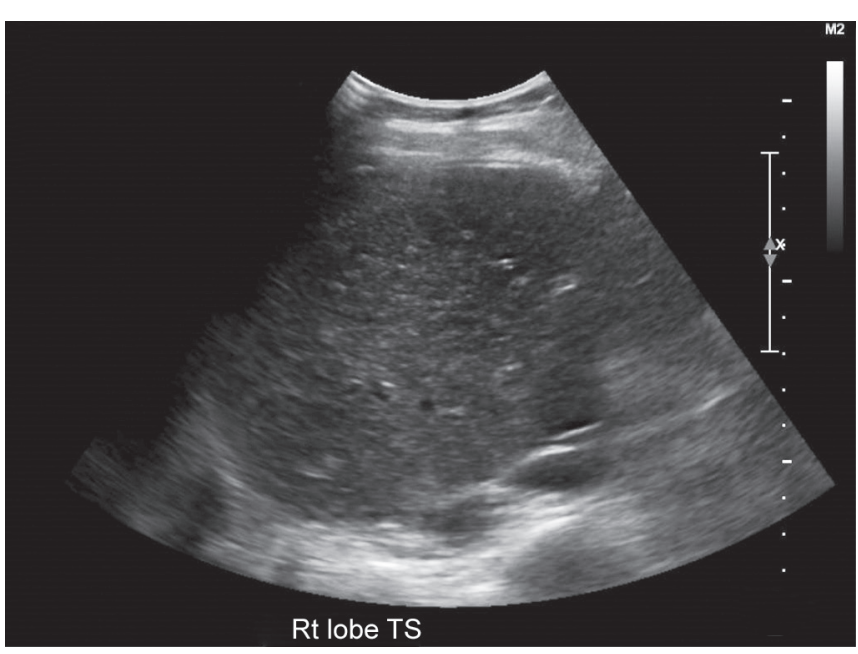

Fig. 1: Hepatic architecture. Mild coarsening of hepatic echotexture is noted on ultrasound scan due to hepatic fibrosis

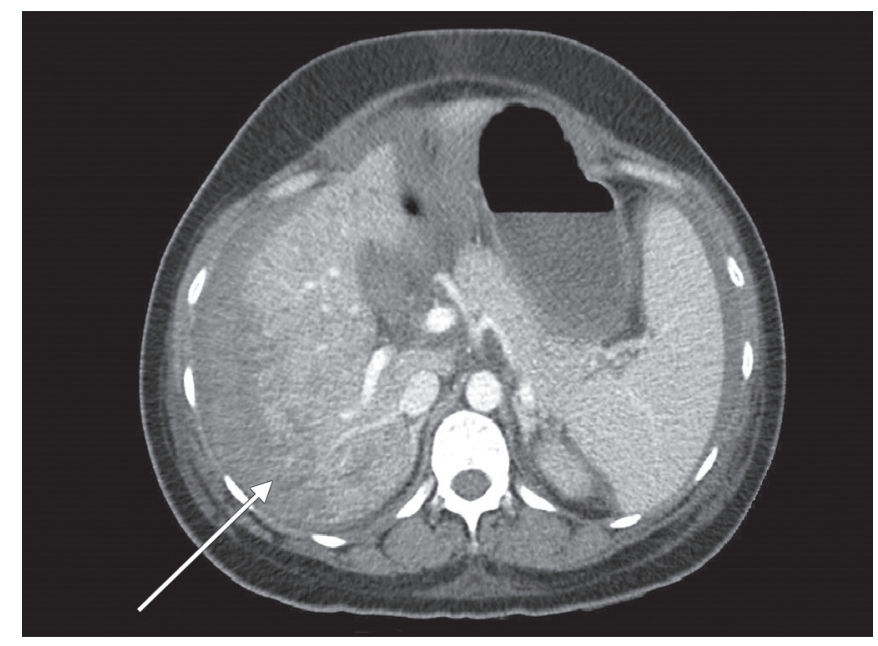

Fig. 2: Subcapsular hematoma. Severe preeclampsia can cause coagulopathies that could lead to subcapsular hematomas as noted on this CT scan image

showed thrombocytopenia, worsening transaminitis, and coagulation derangement. An ultrasound scan of the abdomen showed hepatomegaly, mild coarsening of the hepatic echo texture, increased periportal echogenicity, and diffuse edema of the gallbladder wall that raised the possibility of hepatitis or third spacing with moderate ascites (Fig. 1).

A gastroenterologist's review was sought. Further investigations revealed normal serum lipase, amylase, and hepatitis screen. Random serum glucose $(8 \mathrm{nmol} / \mathrm{L}$, normal range 3.9-6.0) and lactate dehydrogenase (1289 $\mathrm{u} / \mathrm{L}$, normal range 120-260) were elevated and haptoglobin was $<0.10 \mathrm{gm} / \mathrm{L}$ (normal range $0.37-2.70$ ). Peripheral blood smear showed hypochromic microcytic red blood cells, some polychromatic macrocytes, poikilocytes, few target cells, and metamyelocytes. Her liver function and coagulation profile were closely monitored and serially trended for a day during her stay in our institution.

Her epigastric pain worsened, with radiation to her left hypochondrial region along with increasing abdominal distension and abdominal petechial rash. An urgent computed tomography (CT) scan of abdomen and pelvis revealed multiple areas of active extravasation along hepatic capsular surface and a subcapsular hematoma with possible extension into peritoneal cavity. Large amount of ascites with bilateral pleural effusions and atelectasis of lungs was noted (Fig. 2).

Liver function progressively worsened and she developed DIVC [fibrinogen $0.47 \mathrm{gm} / \mathrm{L}$ (normal range 1.80-4.80), activated partial thromboplastin time 52.1 seconds (normal range 29.7-45.6), prothrombin time 26.3 seconds (normal range 11.6-14.2), international normalized ratio 2.47]. Two units of fresh frozen plasma, 1 unit of cryosupernatant, 10 units of cryoprecipitate, and 5 units of packed cell were transfused. 
She was then transferred to the high dependency unit of another tertiary general hospital for further management. Serial hepatic artery angiogram revealed hemoperitoneum and perihepatic hematoma with no signs of active bleeding. She was conservatively managed with close monitoring of hemoglobin, platelets, liver functions, and coagulation profile. She received two more units of packed cell for a drop in hemoglobin from 7.2 to $5.4 \mathrm{gm} /$ $\mathrm{dL}$, which subsequently stabilized at $8 \mathrm{gm} / \mathrm{dL}$ without a need for any further transfusions. Her liver function and platelet counts improved. She was discharged on 14th postnatal day in stable condition.

\section{DISCUSSION}

In total, $70 \%$ of HELLP syndrome occurs in the antenatal period and 20 to $30 \%$ occurs in early postpartum period, with about $10 \%$ occurring in the first week postdelivery. ${ }^{10}$ Patients usually complain of nonspecific symptoms, such as malaise, epigastric/right upper quadrant pain, or nausea/vomiting, mimicking other hepatic conditions, such as acute fatty liver in pregnancy, obstetric cholestasis, and viral hepatitis.

Abercrombie $^{11}$ first described liver hematomas in pregnancy in 1844. Symptoms are nonspecific including acute abdominal/epigastric pain radiating to right shoulder, nausea/vomiting, and abdominal distension that makes early diagnosis challenging. Hemodynamic instability with hypovolemic shock may be present in cases of hepatic rupture.

Rupture of liver is almost always preceded by parenchymal hematoma usually developing in the right lobe of the liver. ${ }^{6}$ Occasionally, hepatic hematomas can be associated with renal hematomas. ${ }^{12}$ Exact pathophysiology and cause of intrahepatic hematoma are unknown. There is a suggestion that it could be secondary to fibrinoid thrombi formed from DIVC in the liver sinusoids causing necrosis and periportal hematoma. ${ }^{3}$ Minor trauma and uncontrolled hypertension may further increase the risk of capsular rupture due to overdistension of the Glisson's capsule.

Diagnosis is aided by ultrasound, CT, or magnetic resonance imaging. In our case, CT scan was done to visualize the entire abdomen to rule out other causes of abdominal distention and pain.

Treatment of liver capsular hematoma with rupture and hemoperitoneum requires a multidisciplinary team involving obstetricians, neonatologists, anesthetists, and gastroenterologists. It requires aggressive fluid resuscitation and infusion of blood products. In patients who are hemodynamically unstable, surgical intervention including drainage, packing, hepatic artery ligation, and partial hepatectomy of affected segment or liver transplant may be required. ${ }^{9}$ In selected cases, they can be managed conservatively with or without transcatheter hepatic artery embolization. Increasingly, conservative treatment is becoming the mainstay of treatment due to a better understanding of pathophysiology of HELLP syndrome and preeclampsia along with advances in diagnostic imaging techniques. Postnatally, it is important to follow the patient up with serial imaging till the hematoma resolves.

Mortality in patients with liver hematomas is most commonly associated with coagulopathy and exsanguination. ${ }^{13,14}$ In our case, though she had coagulopathy, she had a good outcome with conservative management as she responded well to supportive treatment.

Hemoperitoneum secondary to rupture of hepatic hematoma has been reported in the literature before, ${ }^{15-17}$ with surgical treatment being the mainstay of management. Only one case was being conservatively managed.

\section{CONCLUSION}

Prompt recognition with careful clinical examination, laboratory investigations, and imaging, followed by aggressive resuscitation, supportive treatment, and early fetal delivery remains the cornerstone in the management of hemoperitoneum secondary to liver hematoma rupture in patients with severe preeclampsia. In this case, although reaching the diagnosis was initially challenging, due to appropriate supportive treatment, both mother and baby had a good outcome.

\section{CLINICAL SIGNIFICANCE}

Most cases with liver hematomas may lead to hepatic rupture with hemodynamic instability necessitating an aggressive operative approach. However, conservative management with close monitoring of hemodynamic status can be undertaken if the patient is hemodynamically stable with good maternal outcome, as illustrated in this case.

\section{REFERENCES}

1. Weinstein L. Syndrome of hemolysis, elevated liver enzymes, and low platelet count: a severe consequence of hypertension in pregnancy. Am J Obstet Gynecol 1982 Jan;142(2):159-167.

2. Pritchard JA, Weisman R Jr, Ratnoff OD, Vosburgh GJ. Intravascular hemolysis, thrombocytopenia and other hematologic abnormalities associated with severe toxemia of pregnancy. N Engl J Med 1954 Jan;250(3):89-98.

3. Abildgaard U, Heimdal K. Pathogenesis of the syndrome of hemolysis, elevated liver enzymes, and low platelet count (HELLP): a review. Eur J Obstet Gynecol Reprod Biol 2013 Feb;166(2):117-123.

4. Haram K, Svendsen E, Abildgaard U. The HELLP syndrome: clinical issues and management. A Review. BMC Pregnancy Childbirth 2009 Feb;9(1):8. 
5. Haddad B, Barton JR, Livingston JC, Chahine R, Sibai BM. Risk factors for adverse maternal outcomes among women with HELLP (hemolysis, elevated liver enzymes, and low platelet count) syndrome. Am J Obstet Gynecol 2000 Aug;183(2):444-448.

6. Wicke C, Pereira PL, Neeser E, Flesch I, Rodegerdts EA, Becker HD. Subcapsular liver hematoma in HELLP syndrome: evaluation of diagnostic and therapeutic options-a unicenter study. Am J Obstet Gynecol 2004 Jan;190(1):106-112.

7. Barton JR, Sibai BM. Hepatic imaging in HELLP syndrome (hemolysis, elevated liver enzymes, and low platelet count). Am J Obstet Gynecol 1996 Jun;174(6):1820-1827.

8. Nunes JO, Turner MA, Fulcher AS. Abdominal imaging features of HELLP syndrome: a 10-year retrospective review. Am J Roentgenol 2005 Nov;185(5):1205-1210.

9. Grand'Maison S, Sauvé N, Weber F, Dagenais M, Durand M, Mahone M. Hepatic rupture in hemolysis, elevated liver enzymes, low platelets syndrome. Obstetrics \& Gynecology. 2012 Mar 1; 119(3): 617-25.

10. Sibai BM, Ramadan MK, Usta I, Salama M, Mercer BM, Friedman SA. Maternal morbidity and mortality in 442 pregnancies with hemolysis, elevated liver enzymes, and low platelets (HELLP syndrome). Am J Obstet Gynecol 1993 Oct;169(4): 1000-1006.
11. Abercrombie J. Case of hemorrhage of the liver. Lond Med Gaz 1844;34:792-794.

12. Chan $\mathrm{AD}$, Gerscovich EO. Imaging of subcapsular hepatic and renal hematomas in pregnancy complicated by preeclampsia and the HELLP syndrome. J Clin Ultrasound 1999 Jan;27(1): 35-40.

13. Smith LG Jr, Moise KJ Jr, Dildy GA 3rd, Carpenter RJ Jr. Spontaneous rupture of liver during pregnancy: current therapy. Obstet Gynecol 1991 Feb;77(2):171-175.

14. Barton JR, Sibai BM. HELLP and the liver diseases of preeclampsia. Clin Liver Dis 1999 Feb;3(1):31-48.

15. Pavlis T, Aloizos S, Aravosita P, Mystakelli C, Petrochilou D, Dimopoulos N, Gourgiotis S. Diagnosis and surgical management of spontaneous hepatic rupture associated with HELLP syndrome. J Surg Educ 2009 Jun;66(3):163-167.

16. Araujo AC, Leao MD, Nobrega MH, Bezerra PF, Pereira FV, Dantas EM, Azevedo GD, Jeronimo SM. Characteristics and treatment of hepatic rupture caused by HELLP syndrome. Am J Obstet Gynecol 2006 Jul;195(1):129-133.

17. Manas KJ, Welsh JD, Rankin RA, Miller DD. Hepatic hemorrhage without rupture in preeclampsia. N Engl J Med 1985 Feb;312(7):424-426. 